\title{
DEMOGRAPHIC-TOXICOLOGICAL STUDIES RELATED WITH RESISTANCE OF DIFFERENT PESTICIDES ON TETRANYCHUS URTICAE KOCH(ACARI : TETRANYCHIDAE)
}

\author{
SALWA E. NEGM'1, A. A. SALEH'1, A. A. ABD ELHADY1, H. A. BEKHEAT² \\ AND RANIA A.ABD ELWAHAB²
}

1. Pesticide Dept., Fac. of Agric. , Mansoura Univ.

2. Plant Protection Research Institute,ARC, Dokki,Giza

(Manuscript received 28 April 2009)

\begin{abstract}
Demographic bioassays were carried out in the laboratory to assess the demographic responses with gained resistance of Tetranychus urticae Koch to Vertimec, Cypermethrin, Methomyl and Malathion at two sublethal concentrations(LC50 and LC25) through 40 generations. Life table data were used in each sublethal treatment of parents , F2, F6 ,F10 , F20 , F30 and F40. Life cycle durations ranged from 30.73 to 16.05 days at LC50 and from 23.84 to 11.87 days at LC25 of tested pesticides,resp. Life span durations ranged from $21.71 \%$ to $-28.12 \%$ at LC50 and from $17.84 \%$ to $30.96 \%$ at LC25 of tested pesticides,comparing with the check. Intrinsic rate of increase $(\mathrm{Rm})$ and two population growth rates(fecundity $\lambda$ and vitality $\lambda$ )for T.urticae (hereafter given in units $\lambda$ /day)were estimated and discussed
\end{abstract}

\section{INTRODUCTION}

Demographic analysis appeared to be an ideal method of evaluating pesticide effects, because it combined both lethal and sublethal pesticides effects.In these demographic experiments, individuals or groups were exposed to a series of constant concentrations and observations were made on various life-history events,such as life span (Stark and Rangus, 1994), development rates (Vinson,1974), fecundity (Stark et al.,1992), and longevity (Stark and Banks, 2003). Demographic-toxicology is an ecotoxicological technique that incorporates life table parameters in the context of toxicology (Levin et al.,1996). By combining lethal and sublethal effects, it is possible to predict the total effects of pesticides at any population level.

It is an importa nt advantage inherent in using the demographic toxicological approach over traditional lethal concentration estimates.Not only was total measurements of obtained toxic effects, but other interactions in short-term toxicity tests could be evaluated. Results of several studies had indicated that sublethal effects could be very subtle and affected populations at concentrations lower than traditional concentration response curve.(Stark and Banks, 2003).

The present study aimed to measure the effect of sublethal tested pesticides on T.urticae, with combination to resistance which gained through 40 generations . 


\section{MATERIALS AND METHODS}

Pesticides and Chemicals: The formulated pesticides used for leaf-dip bioassays were Vertimec (Averrmectines),Cypermethrin(Pyrethroids),Methomyl(Carbamates) and Malathion(Organophosphates s).parasympathomimetic

Maintenance of Tetranychus urticae: Colonies of the spider mite, T.urticae were reared under laboratory conditions $\left(25 \pm 2^{\circ} \mathrm{C}\right.$, and $\left.60 \pm 5 \% \mathrm{RH}\right)$ at Plant Protection Research Institute Branch, Dakahlia Governorate. Mites were reared on leaves of castor oil ,these leaves were cleaned and placed on moist cotton wool pad in Petridishes.The beginning of these colonies were isolated from infested leaves of castor oil, left for one year under the previous conditions in order to get a homogenous and sensitive colony. Spider mites were transferred to the leaves by the aid of the fine camel's hair brush,adding water was done twice daily to prevent escaping of T.urticae individuals.

\section{The Assessment of the chronic toxicity of tested pesticides against}

T.urticae: These studies were conducted to evaluate the comparative chronic toxicity of tested pesticides at LC25 and LC50 from the parents generation to $40^{\text {th }}$ generation, which were reared under selection pressure.

Three leaf -discs were considered as three replicates for each concentration. Leaf - dipping technique was used as described by Dittrich(1962).Leaf-discs were placed on moisted cotton pad in petri-dishes after treatments .30adult females of parents and then $2^{\text {nd }}, 4^{\text {th }}, 10^{\text {th }}, 20^{\text {th }}, 30^{\text {th }}$ and $40^{\text {th }}$ generations, were chosen randomly and transferred with 30adult males to leaf -discs by the aid of the camel's hair brush using stereomicroscope. Treated and untreated individuals allowed to lay eggs for 24 hours according to Yassin(1997)and Abd Elwahab(2003), then adult females were removed . Eggs were left for hatching and the duration periods of developmental stages were recorded for T.urticae progeny every two generations and treated with the newly values of LC25 and LC50 of each tested pesticide.

Total immature durations, life cycle, longevity, life span, survival probability, fecundity, vitality and intrinsic rate of increase were recorded. Hatchability and sterility were also recorded. All these data were analyzed up on demographic assay according to Kim et al. (2004).

\section{RESULTS AND DISCUSSION}

Assessments of the chronic toxicity of Vertimec,Cypermethrin,Methomyl and Malathion on some biometric measurements of T.urticae through 40 generations were shown in Tables (1 to 7 ).For this purpose,four tested compounds used in the 
selection pressure by two sublethal concentrations (LC50 and LC25) were used to gain resistant populations of T.urticae to each pesticide at two levels.At the start of this work,adult females were allowed to laid eggs for 24 hours.The demographictoxicological parameters were studied upon Kim et al.(2004) for parents, $2^{\text {nd }}, 6^{\text {th }}, 10^{\text {th }}$, $20^{\text {th }}, 30^{\text {th }}$ and $40^{\text {th }}$ generations.

\section{Effect on incubation period}

Table ( 1 ) showed that incubation periods were affected by the tested compounds. It was clear that LC50 of tested pesticides prolonged the incubation period than that of the control,through the experimental generations.Incubation periods affected by Vertimec LC50 recorded (6.94, 6.25, 5.92, 5.32, 5.14 and 5.77)days for $F 2$,F6 ,F10 , F20 ,F30 and F40, resp. Cypermethrin LC50 showed (6.73, 6.17, 5.83, $5.24,5.20$ and 5.32)days, resp.,then Methomyl LC50 (5.25, 5.14, 5.07, $4.75,4.60$ and 5.32 ) days, resp.While the incubation periods with malathion were (5.43, 5.23, 5.10, 5.02, 4.73 and 4.55) days,resp.

Variations are shown in case of LC25 treatments with tested pesticides.Some treatments caused prolongation and others caused shortening incubation periods affected by Vertimec LC25 recorded ( 5.30, 5.42, 5.29, 5.13, 4.53 and 4.37)days ,resp.,followed by Cypermethrin LC25( 5.22, 5.15, 5.04, 4.97,4.48 and 4.21)days,resp.,then Methomyl LC25 recorded ( 4.97, 4.81, 4.33, 4.12, 3.98 and 3.67)days,resp. While with Malathion ,the incubation periods were $(5.20,5.09,4.87$, $4.62,4.25$ and 3.97) days,resp. On the other hand ,incubation period of T.urticae in the control was 4.30 days.

\section{Effect on total immature durations}

Data in Table ( 2 ) showed that immature durations affected by Vertimec LC50 recorded $(88.66,75.57,62.49,41.87,30.06$ and 9.10$) \%$ for F2 , F6 , F10 , F20 , F30 and F40, resp.,followed by Cypermethrin LC50 (22.82, 21.26, 19.78, 16.15 , 15.68 and 13.10 ) \%, resp.,then Methomyl LC50 ( $20.49,19.53,17.96,15.83,13.51$ and 11.86 ) \%,resp.While in the case of Malathion ,the immature durations were $(20.86,19.33,17.90,16.28,13.38$ and 11.50$) \%$,respectively,comparing with the control. Variations are shown in case of LC25 treatments with tested pesticides.Some treatments caused prolongation and others caused shortening incubation periods affected by Vertimec LC25 recorded ( $17.27,17.79,15.84,14.04,12.35$ and 10.64 ) days , resp.,followed by Cypermethrin LC25( $18.62,17.09,14.97,13.22,11.29$ and 9.79 ) days,resp.,then Methomyl LC25 recorded ( $17.17,16.57,14.38,9.23,10.49$ and 8.20 ) days,resp. While in the case of Malathion, the immature periods were (17.43, $16.68,14.45,12.94,10.66$ and 9.3 ) days,resp. On the other hand ,immature period of T.urticae in the control was 12.61 days. 


\section{Effect on life cycle durations}

Table ( 3 ) showed that life cycle durations were affected by the tested compounds .It was clear that most LC50's of tested pesticides prolonged the incubation period than that of the control,through experimental generations.Life cycle durations affected by Vertimec LC50 recorded ( 30.73 ,28.39 26.41, 23.21, 21.54 and 19.64 ) days for $F 2$, F6 ,F10 ,F20 ,F30 and F40, resp.,followed by Cypermethrin LC50 ( $29.55,27.43,25.61,21.39,24.27$ and 18.42 ) days, resp.,then Methomyl LC50 ( $25.74,24.67,23.03,20.58,18.11$ and 16.25 ) days,resp.While in case of Malathion ,the life cycle durations were $26.2,24.56,23.00,21.30,18.11$ and 16.05) days, resp. Variations are shown in case of LC25 treatments with tested pesticides.Some treatments caused prolongation and least of them caused shortening incubation periods affected by Vertimec LC25 recorded ( $22.57,23.21,21.13,19.17$ ， 16.88 and 15.01 )days ,resp.Followed by Cypermethrin LC25(23.84, 22.24, 20.01 , 18.19 15.77 and 14.00 )days,resp.,then Methomyl LC25 recorded ( $22.14,21.38$, $18.71,13.35,14.47$ and 11.87 )days,resp. While in the case of Malathion ,the immature periods were $(22.63,21.77,19.32,17.56,14.91$ and 13.27$)$ days,resp. On the other hand, life cycle period of T.urticae in the control was 17.31 days.

\section{Effect on longevity durations}

Table ( 4 ) showed that longevity durations were affected by the tested compounds. It was clear that dereliction of longevity durations caused by all LC50's of tested pesticides were affected especially with Vertimec LC50 ( $-44.59,-47.30,-55$, $-62.5,-73.18$ and -74.59$) \%$ for $F 2, F 6, F 10, F 20$,F30 and F40, resp,followed by Cypermethrin LC50 ( $-41.55,-44.59,-50.61,-58.18,-62.64$ and -67.77$) \%$, resp.,then Methomyl LC50 (-21.62, $-28.51,-32.43,-40.27,-44.46$ and -53.85$) \%$, resp.While in the case of Malathion, the longevity durations were $(-18.65,-22.09$, $28.04,-31.82,-37.5$ and -45.14$) \%$, resp.

Considering the effects of LC25 treatments with tested pesticides, they were less pronounced than LC50's. The most affected longevity durations caused by Vertimec LC25 which recorded ( - $-16.96,-22.43,-27.84,-36.55$, 46.35 and $-51.62 \%$ ) ,resp. ,followed by Cypermethrin LC25 $(-18.72,-19.05,-30.34,-32.84$ and $-39.19 \%)$, resp., then Methomyl LC25 recorded (-1.28,-6.22,-10.41,-15,-19.12 and $-18.78 \%$ ), resp. While in the case of Malathion ,the longevity durations have significant variance.Longevity of $\mathrm{F} 2$ was prolonged than control with $2.77 \%$,otherwise the rest of generations' longevity decreased with $(-1.28,-4.80,-9.86,-13.78$ and $-18.78 \%)$ resp.,of $F 4$,F10,F20 ,F30 and F40,resp. On the other hand ,longevity period of T.urticae in the control was 14.8 days. 


\section{Effect on life span durations}

Data in Table ( 5 ), showed that LC50 of tested pesticides varied from prolongation and shortening of life span durations, of each selected generation . Vertimec LC50 recorded $(21.24,21.71,2.99,-10.43,-20.55$ and $-27.13 \%)$ for F2, F6, F10 F20 ,F30 and F40,resp.,followed by Cypermethrin LC50 (18.97,10.96,2.52,$14.11,48.86$ and $-27.78 \%$ ), resp.,for the same arrangement of selected generations , then Methomyl LC50 $(16.29,9.78,2.87,-8.38,-18.00$ and $-28.12 \%)$,resp. Malathion LC50 showed $(19.09,12.39,4.80,-2.24,-14.79$ and $-24.73 \%)$, resp.comparing with the control life span.

In the same trend, Table ( 5 ),showed the effects of LC25 of tested pesticides which varied from prolongation and shortening of life span durations, of each selected generation . Vertimec LC25 recorded ( 8.56,8.03,-0.93,-11.06,-22.70 and $-30.96 \%$ ) for F2, F6 ,F10 ,F20 ,F30 and F40,resp., followed by Cypermethrin LC25 (11.71,6.57,$3.46,-11.24,-19.93$ and $-28.37 \%$ ), resp.,for the same arrangement of selected generations ,then Methomyl LC25 (14.45,9.81,-0.44,-19.25,-17.66 and -27.56 \%), resp., and finally the least effective pesticides, Malathion LC25 $(17.84,13.30,4.05,-$ $1.43,-13.83$ and $-21.24 \%$ ), resp.comparing with the control life span (32.11) days.

\section{Effect on fecundity $\lambda$}

The population growth rate (fecundity $\lambda$ ) of T.urticae generations affected by tested compounds was listed in Table ( 6 )

Lowering of fecundity was occurred through generations treated with LC50,s of tested pesticides. Fecundity $\lambda$ upon Vertimec LC50 recorded $(0.52,0.37,0.24$, $0.21,0.31$ and $0.11 \lambda$ /day ) for $F 2, F 6, F 10, F 20, F 30$ and $F 40$,resp.,followed by that upon Cypermethrin LC50 ( $0.63,0.50,0.47,0.42,0.32$ and $0.20 \lambda /$ day ), resp. While , fecundity $\lambda$ upon Methomyl LC50 recorded ( $0.72,0.66,0.54,0.50,0.40$ and $0.31 \lambda /$ day),resp.In the same trend, the case of fecundity $\lambda$ upon Malathion LC50 values were ( $0.81,0.74,0.60,0.57,0.47$ and $0.24 \lambda$ /day ),resp. The fecundity $\lambda s$ of all populations affected by tested pesticides LC50's through forty generations were still $<1$,indicating a declining in all populations under selection pressures.

At the same way, continuous fecundity reduction upon LC25's of tested compounds through generations of T.urticae as shown in Table ( 6 ). Fecundity upon Vertimec LC25 recorded ( $0.64,0.60,0.50,0.32,0.24$ and $0.19 \mathrm{~N} /$ day) for F2 ,F6 , F10, F20 ,F30 and F40, resp. ,followed by that upon Cypermethrin LC25 $(0.70,0.63$, $0.52,0.47,0.39$ and $0.24 \lambda /$ day), resp. While, fecundity upon Methomyl LC25 recorded $(1.34,1.27,0.98,0.82,0.73$ and $0.50 \lambda /$ day $)$, resp.In the same trend , fecundity with Malathion LC25 values were ( $1.89,1.53,1.17,0.84,0.52$ and 0.44 $\lambda /$ day), resp. comparing with the control fecundity (5.64 $\lambda /$ day). 
Fecundity $\lambda s$ of all populations affected by Vertimec and Cypermethrin LC25's through forty generations were still $<1$, indicating a decline in all populations under selection pressures.On the other hand,Methomyl and Malathion LC25's caused fecundity $>1$ in (F2 and F6) and (F2,F6 and F10),resp. indicating that the populations were in growth, while through rest generations, fecundity values were $<1$,indicating that the populations were in a decline situation.

\section{Effect on vitality $\lambda$}

The population growth rate (vitality $\lambda$ ) of T.urticae generations affected by tested compounds (hereafter given in units per day)and their bootstrap-estimated $95 \%$ confidence limits for acaricides treatments at two sublethal concentrations are listed in Table (6 ).

Table ( 6 ), showed that vitality of T.urticae was affected by LC50 of the tested compounds . Lessening of vitality was occurred through generations. Vitality upon Vertimec LC50 recorded $(0.31,0.25,0.19,0.14,0.10$ and $0.02 \lambda /$ day $)$ for F2 ,F6, $\mathrm{F} 10$, F20 , F30 and F40 ,resp.Followed by that upon Cypermethrin LC50 ( 0.53 , $0.40,0.29,0.18,0.15$ and $0.13 \lambda$ /day ), resp. While ,vitality upon Methomyl LC50 recorded ( $0.59,0.43,0.31,0.27,0.22$ and $0.19 \lambda$ /day),resp.In the same trend, with Malathion LC50 values were ( $0.64,0.51,0.47,0.32,0.25$ and $0.21 \lambda$ /day),resp. From previous data, the vitality $\lambda s$ of all populations affected by tested pesticides LC50's through forty generations were still $<1$, indicating a declining in all populations under selection pressures.

At the same way, a continuous reduction of vitality upon LC25's of tested compounds through generations of T.urticae is shown in Table ( 6 ). Vitality upon Vertimec LC25 recorded $(0.42,0.38,0.25,0.18,0.12$ and $0.07 \mathrm{~N} /$ day $)$ for F2，F6 , $F 10, F 20, F 30$ and F40, resp.

In the case of Cypermethrin LC25, it showed $(0.60,0.52,0.39,0.25,0.19$ and $0.16 \mathrm{~N} /$ day $)$, resp. While, vitality upon Methomyl LC25 recorded $(0.70,0.63,0.52$, $0.44,0.28$ and $0.21 \mathrm{~N} /$ day ), resp.In the same trend, the case of fecundity upon Malathion LC25 values showed $(0.79,0.73,0.58,0.40,0.35$ and $0.29 \lambda /$ day),resp. comparing with the control vitality (4.97 $\lambda /$ day). Reviewing the previous data, it could be proved that the vitality $\lambda s$ of all populations affected by tested pesticides LC25's through forty generations were continued $<1$, indicating a declining in all populations under selection pressures.

Relative reduction of vitality (R.R.vitality) showed in Table ( 6 ), were varied in its values through generations depending on the tested pesticide. R.R.vitality upon Vertimec LC50 recorded $(0.40,0.32,0.21,0.33,0.68$ and $0.82 \lambda)$ for F2 , F6 , F10 , F20, F30 and F40, resp.,followed by that upon Cypermethrin LC50 $(0.16,0.20,0.38$, 
$0.57,0.53$ and $0.35 \lambda$ ), resp. While, R.R.vitality upon Methomyl LC50 recorded $(0.18,0.35,0.43,0.46,0.60$ and $0.39 \lambda)$, resp.In the same trend, the case of R.R.vitality upon Malathion LC50 values were $(0.19,0.31,0.22,0.44,0.46$ and 0.13 $\lambda)$, resp. Therefore, it could be concluded that Vertimec had a more severe effect on development of T.urticae resistant populations than other pesticides. Concerning Relative reduction of vitality (R. R. vitality) data in Table ( 6 ) showed that depending on LC25's of the tested pesticide. R.R.vitality upon Vertimec LC25 recorded ( 0.34 , $0.37,0.50,0.44,0.50$ an $0.63 d \lambda$ ) for $F 2, F 6, F 10, F 20, F 30$ and $F 40$ ,resp.,followed by that upon Cypermethrin LC25 ( $0.14,0.17,0.25,0.47,0.51$ and $0.33 \lambda)$, resp. While,R.R.vitality with Methomyl LC25 recorded ( $0.48,0.50,0.47$, $0.46,0.62$ and $0.58 \lambda$ ), resp.In the same trend, the case of R.R.vitality upon Malathion LC25 values were $(0.58,0.52,0.50,0.52,0.34$ and $0.34 \lambda)$,resp. In case of R.R.vitality of control, it was the least of all previous treatments values recording $(0.12 \lambda)$. The most superior effective compound category was swinging between Vertimec LC25, which had severe efficacy on T.urticae (F30 and F40), and Malathion LC25, which was effective on earlier generations developments.

\section{Effect on intrinsic rate of increase ( $\mathrm{Rm})$}

The intrinsic rate of increase $(\mathrm{Rm})$ is a measurement of the ability of a population to increase logarithmically.Positive values of $\mathrm{Rm}$ indicte exponential population increase, $\mathrm{Rm}$ equal to zero indicates that the population is stable, and negative values of $\mathrm{Rm}$ indicate that the population is declining exponentially and heading toward extinction. (Carey,1993).

The intrinsic rate of increase (Rm) values of T.urticae was affected by LC50 of the tested compounds. All values were negative as shown in Table ( 7 ) through experimental generations.

Rm upon Vertimec LC50 recorded $(-0.28,-0.43,-0.68,-.051$ and $-0.96 \mathrm{~d}-1)$ for $F 2$, F6 , F10 , F20 , F30 and F40 ,resp.,followed with Cypermethrin LC50 ( -0.20,$0.30,-0.33,-0.38,-0.50$ and $-0.70 \mathrm{~d}-1)$, Methomyl LC50 recorded $(-0.14,-0.18$,$0.27,-0.30,-0.40$ and $-0.51 \mathrm{~d}-1)$, resp, and Rm upon Malathion LC50 values were $(-0.90,-0.13,-0.22,-0.24,-0.33$ and $-0.62 d-1)$, resp. From previous data ,the Rm 's of all populations indicating that population rate was declined.

Rm's upon LC25's of tested compounds through generations of T.urticae were shown in Table (7). Rm depending on Vertimec LC25 and Cypermethrin LC25 recorded negative values $(-0.19,-0.22,--0.30,-0.50,-0.62$ and $-0.72 \mathrm{~d}-1)$ and $(-0.15$, $-0.20,-0.28,-0.33,-0.41$ and $-0.62 \mathrm{~d}-1$ ) resp., for F2 , F6 , F10 , F20, F30 and F40, resp. Those data showed declining population growth rate as a direct result of resistance. While ,Rm's of F2 and F6 treated with Methomyl LC25 recorded positive 
values ( 0.12 and $0.10 \mathrm{~d}-1)$ resp.,but the rest generations treated with the same pesticide at the same sublethal concentrations recorded negative values $(-0.009,-0.09$, -0.14 and $-0.30 \mathrm{~d}-1)$ resp. In the same trend, the case of Rm's upon Malathion LC25 values were $(0.28,0.18$ and $0.07 \mathrm{~d}-1)$, resp. of $F 2, F 6$ and F10,resp. but the rest generations treated with the same pesticide at the same sublethal concentrations recorded negative values $(-0.08,-0.28$ and $-0.36 \mathrm{~d}-1)$ resp.

Previous data meant that populations were increased first, then they were declined as the result of increasing resistance ratio because of continuous chronic toxicity. On the other hand,Rm value of the control population was $(0.75 \mathrm{~d}-1)$.

Most investigations had focused primarily on the immediate contact mortality-toxicity of the pesticides.The characterization of differential susceptibility among life stages was critical in the assessment of pesticides on target species, because it could significantly alter the age distribution and demographic characteristics of exposed mite populations(Stark and Wennergen,1995).The present study showed contribution of T.urticae resistance to tested pesticides in demographic -toxicology depending on sublethal concentrations on biological parameters through assayed generations. Most tested compounds at LC50 and LC25 exhibited a large declines in Rm's. Two different scales of population level endpoint [population growth rates(fecundity $\lambda$ and vitality $\lambda)$ ]were adopted to compare the total acaricidal activities of tested compounds on T.urticae at two sublethal concentrations and to get an accurate image of resistance evolution through generations. The differenc between the fecundity and the vitality resulted from the different effects of the pesticides on egg viabilities.(Kim et al.2004).In other words, fecundity $\lambda$ could be calculated with hatch rate while vitality could be calculated without hatch rate. Therefore, fecundity $\lambda$ values were supposed to be always higher than vitality $\lambda$ at the same level of sublethal concentration. Relative Reduction (RR) of vitality $\lambda$ of all treatments over generations were $>R R$ of vitality of control population, it can be noted that all treatments contributed in resistance, which affected eggs fertility at the population growth levels.

Beside that the demographic effects of pesticides could be assessed on biological as done by Menelaos and Nicholas (2009).They demonstrated that simultaneous testing of the demographic effects of pesticides on pests and natural enemies was essential for a full assessment of pesticide impacts on biological control. Kavousi et al.(2009) studied the life tables of $T$. urticae on leaf discs and whole leaves of the common bean plant, Phaseolus vulgaris L. Data were analyzed based on the age-stage, sexes life table theory. 
DEMOGRAPHIC-TOXICOLOGICAL STUDIES RELATED WITH RESISTANCE OF DIFFERENT PESTICIDES ON TETRANYCHUS URTICAE KOCH(ACARI : TETRANYCHIDAE) 
DEMOGRAPHIC-TOXICOLOGICAL STUDIES RELATED WITH RESISTANCE OF DIFFERENT PESTICIDES ON TETRANYCHUS URTICAE KOCH(ACARI : TETRANYCHIDAE) 


\section{REFERENCES}

1. Abd Elwahab, R. A. 2003.Studies on some chemical compounds against some tetranychid mite species.M.Sc.Thesies.,Pesticides Dept.,Fac.of Agric.,Mansoura Univ., Egypt.131p.

2. Carey, J. R.1993. Applied demography for biologists.Oxford,UK:Oxford Univ.Press.

3. Dittrich, V. 1962. Acomparative study of toxicological test methods on a population of the two-spotted spider mite, T.urticae.J.Econ.Entomol.55:633-648.

4. Hayashi, A.1983. History,present status and management of insecticide resistance. Pest Reistance to Pesticides Soft Sciences,Tokyo,pp.31-53.

5. Kavousi, A., H. Chi, K. Talebi, A. Bandani, A. Ashouri and V. Naveh. 2009.Demographic Traits of Tetranychus urticae (Acari: Tetranychidae) on Leaf Discs and Whole Leaves .J. Econ. Entomol. 102 ( 2): 595-601.

6. Kim, M., D. Shin, E. Shu and K. Cho. 2004. An assessment of the chronic toxicity of fenopyroximate and pyridaben to Tetranychus urticae using a demographic bioassay.Appl.Entomol.Zool.39(3):401-409.

7. Levin, L. H. Caswell, T. Bridges, C. Dibacco, D. Cabrera and G. Palia. 1996. Demogarphic-respononses of estuarine ppolychaetes to pollutants : life tableresponse experiments. Ecol. Appl. 6:1295-1313.

8. Menelaos C. S. and J. M. Nicholas. 2009. Demographic effects of pesticides on biological control of Pacific spider mite (Tetranychus pacificus) by the western predatory mite (Galendromus occidentalis). Biol. Control.48(3):267-273.

9. Stark, J. D. and J. E. Banks. 2003. Population-level effects of pesticides and other toxicants on arthropods.Annu.Rev.Entomol.48:L505-519.

10. Stark, J. D. and T. Rangus. 1994.Lethal and sublethal effects of the neem insecticide,Margosan-O, on the pea aphid. Pestic. Sci. 41:155-160.

11. Stark, J. D., R. I. Vargas, R. H. Messing and M. Purcell. 1992.Effects of cyromazine and diazinon on three economically important Hawaiian tephritid fruit flies (Diptera:Tephritidae) and their endoparasitoids (Hymenoptera:Braconidae).J. Econ. Entomol.85:1687-1694.

12. Stark, J. D. and U. Wennergren. 1995. Can population effects of pesticides be predicted from demographic toxicological studies?.J.Econ.Entomol.88:1089-1096.

13. Vinson , S. B. 1974. Effect of an insect growth regulator on two parasitoids developing from treated tobacco budworm larvae. J. Econ. Entomol. 67:335-337.

14. Yassin, E. A. 1997. Biological and ecological studies on some phytophagous mites associated with some field crops.M.Sc.Thesies,Entomol.Dept.,Sci. Faculty,Cairo Univ., Egypt.152p. 
دراسات سميه ديموجرافية متعلقة بالمقاومة

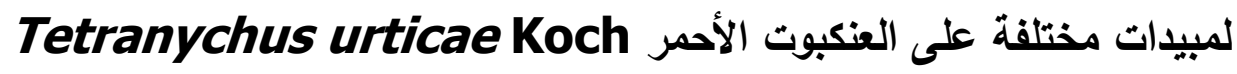
(Acari:Tetranychidae)

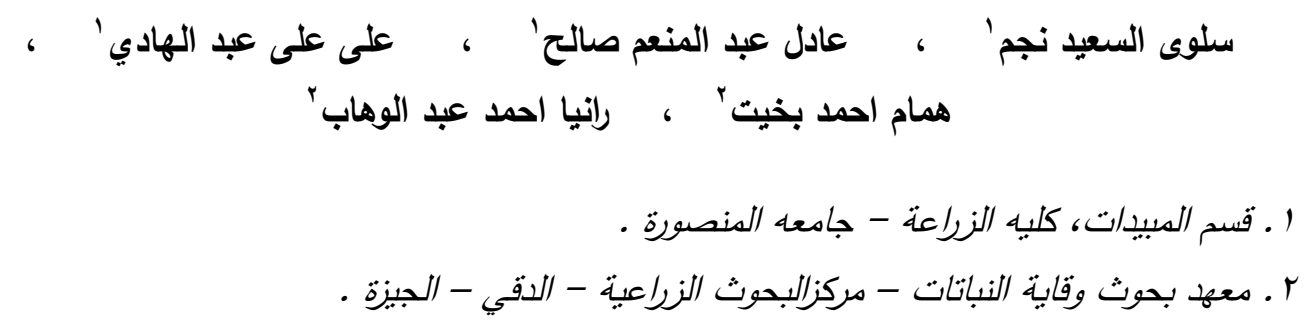
T.urticae تم تقديرها و مناقشتها. 